Discrete Comput Geom 32:309-315 (2004)

DOI: $10.1007 / \mathrm{s} 00454-004-1097-3$

\title{
Unflippable Tetrahedral Complexes
}

\author{
Randall Dougherty, ${ }^{1}$ Vance Faber, ${ }^{2}$ and Michael Murphy ${ }^{3}$ \\ ${ }^{1}$ San Diego, CA, USA \\ rdough@mailhost.ccrwest.org \\ ${ }^{2} 33740$ NE 84th Pl., \\ Carnation, WA 98014, USA \\ vance@mappingscience.com \\ ${ }^{3}$ Fusion Numerics Inc., 1320 Pearl Street, \\ Boulder, CO 80302, USA \\ mike@fusionnumerics.com
}

\begin{abstract}
We present a 16-vertex tetrahedralization of $\mathbb{S}^{3}$ (the 3-sphere) for which no topological bistellar flip other than a 1-to-4 flip (i.e., a vertex insertion) is possible. This answers a question of Altshuler et al. which asked if any two $n$-vertex tetrahedralizations of $\mathbb{S}^{3}$ are connected by a sequence of 2-to-3 and 3-to-2 flips. The corresponding geometric question is whether two tetrahedralizations of a finite point set $S$ in $\mathbb{R}^{3}$ in "general position" are always related via a sequence of geometric 2-to-3 and 3-to-2 flips. Unfortunately, we show that this topologically unflippable complex and others with its properties cannot be geometrically realized in $\mathbb{R}^{3}$.
\end{abstract}

\section{Introduction}

Bistellar flips (also known as "Topological flips" or "Pachner moves") are operations which transform one simplicial decomposition of a manifold to another simplicial decomposition of the same manifold. Definitions of geometric and topological bistellar flips can be found in Section 2. Due to their simplicity and power, these operations have enjoyed many uses in geometric and topological computations. Among the most famous appearances of geometric bistellar flips in Computational Geometry are: Lawson's algorithm for transforming an arbitrary triangulation of a planar point set to its Delaunay triangulation [La]; the improved analysis of the incremental version by Guibas et al. [GKS]; and the generalization of the incremental algorithm to arbitrary dimension by 
Edelsbrunner and Shah [ES]. In a similar vein, bistellar flips can form the basis of algorithms to delete [De] and move a point in a Delaunay triangulation of any dimension while maintaining the triangulation. In Mesh Generation, bistellar flips are often used to improve the quality of elements in two- and three-dimensional unstructured FiniteElement meshes $\left[\mathrm{CDE}^{+}\right]$. In Computational Topology, bistellar flips are used to reduce a simplicial complex to a smaller, topologically equivalent one, giving a useful method to recognize a manifold [BL]. In Mathematical Physics, bistellar moves are used for simplicial quantum gravity simulations $[\mathrm{ACM}]$.

This paper is concerned with Flip graph connectivity questions in both the geometric and topological setting. The Flip graph of a set of simplicial complexes $N$ is defined as follows. Let $N$ be the vertices. Place an edge between two complexes $\mu, v$ in $N$ if there exists a bistellar move which transforms $\mu$ to $v$. In particular we are interested in two analogous questions:

1. Is the flip graph connected when $N$ is the set of all geometric tetrahedralizations of a fixed point set $S$ in $\mathbb{R}^{3}$ ?

2. Is the flip graph connected when $N$ is the set of all topological tetrahedralizations of $\mathbb{S}^{3}$ on $n$ vertices?

When considering the first question, we will assume the points of $S$ are in general position. When considering both questions, note that we cannot allow bistellar flips which change the number of verticies. Thus, we will consider only 2-to-3 and 3-to-2 flips in either case. We now describe the motivation for these questions and review what is known about them.

\subsection{Geometric Flip-Graph Connectivity Questions}

The flip-graph connectivity of the geometric tetrahedralizations of a set of points in $\mathbb{R}^{3}$ is an important open question. The question probably arose from attempts to generalize Lawson's algorithm for computing the Delaunay triangulation of a set of points $S$ in the plane to three and higher dimensions. Briefly, given a triangulation of a set of $n$ planar points, $S$, Lawson showed how to compute the Delaunay triangulation of $S$ using a sequence of at most $O\left(n^{2}\right)$ geometric bistellar flips. Thus, it is clear that the flip graph of triangulations of a planar point set is connected. However, the three-dimensional generalization Lawson's algorithm-perform bistellar flips to replace pairs (triples) of tetrahedra which are not locally Delaunay with triples (pairs) that are - can dead-end in a tetrahedralization which is not Delaunay [Jo]. It is natural to wonder if a better selected sequence of bistellar flips would lead to the Delaunay tetrahedralization; doing so raises the flip-graph connectivity question we address (see [Jo] and [EPW]).

de Lorea $[\mathrm{dL}]$ has examples of point sets in $\mathbb{R}^{3}$ whose flip graphs are of "low connectivity," suggesting that there may be others which are disconnected. Further, there are point sets in $\mathbb{R}^{6}$ for which no geometric bistellar move is possible other than a point insertion [Sa]. In contrast, it is known that the flip graph defined over the regular tetrahedralizations of a point set is connected [BS]; moreover, an efficient algorithm is known $\left[\mathrm{CDE}^{+}\right]$. 


\subsection{Topological Bistellar Flips}

A classic theorem in Combinatorial Topology due to Pachner implies the following: If $N$ is the set of all simplicial decompositions of a particular piecewise-linear manifold, the flip graph is connected. Moreover, two simplicial decompositions cannot be connected by a sequence of bistellar flips if they are not piecewise-linearly homeomorphic. Pachner's theorem is given in $[\mathrm{Pa}]$; a more accessible proof can be found in [Li]. Nevertheless, Pachner's theorem says nothing about the number of vertices which may have to be added to transform one simplicial decomposition to another.

Thus, one might ask if the flip graphs of $n$-vertex triangulations of $d$-spheres are connected, if only to gain insight into the geometric problem. In the two-dimensional case an affirmative result has been known for many years [Wa]. In [ABS] Altshuler et al. asked whether all $n$-vertex tetrahedralizations of $\mathbb{S}^{3}$ are related by bistellar operations which preserve the number of vertices. We show his question, our Question 2, is false in Section 3 where we exhibit a 16-vertex tetrahedralization of $\mathbb{S}^{3}$ for which no bistellar move which preserves the number of vertices is possible. An interesting property of tetrahedralizations of $\mathbb{S}^{3}$ is that not all of them have straight-line geometric realizations in $\mathbb{R}^{3}$, unlike triangulations of $\mathbb{S}^{2}$. If they did, our counterexample would resolve the flip-graph connectivity question for tetrahedralization of point sets. Unfortunately, our counterexample does not admit such a realization, which we demonstrate.

\section{Bistellar Flips}

In this section we enumerate the two- and three-dimensional bistellar flips. An algebraic definition of a bistellar flip, independent of the dimension of the simplicial complex, can be found in [Li].

In two dimensions, a bistellar flip is either a vertex insertion (type 1-to-3), a vertex deletion (type 3-to-1), or an edge flip (type 2-to-2), as illustrated in Fig. 1. Notice that a 1-to-3 flip is always possible: pick a triangle and add a new vertex. However, a 3-to-1 flip to remove a vertex $v$ can occur only if $v$ has exactly three incident edges. Further, there are restrictions on the "flippability" of an edge. Suppose edge $(a, b)$ has two incident triangles $(a, b, c)$ and $(a, b, d)$. We say $(a, b)$ is topologically unflippable if the edge $(c, d)$ is also present in the triangulation. In a geometric setting, an edge $(a, b)$ can be geometrically unflippable even if it is topologically flippable. See Fig. 2. For this reason we distinguish between a geometric bistellar flip and a topological bistellar flip.

In three dimensions, a bistellar flip is either a 1-to-4, 4-to-1, 2-to-3, or a 3-to-2 flip, corresponding to a vertex insertion, vertex removal, face-to-edge flip, or an edge-to-

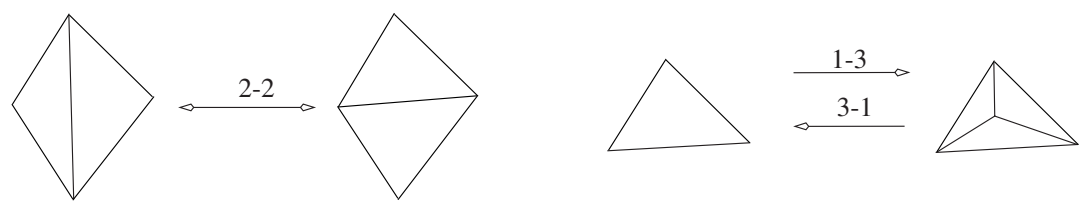

Fig. 1. Bistellar flips in two dimensions. 


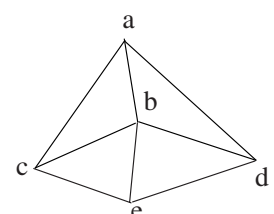

(a)

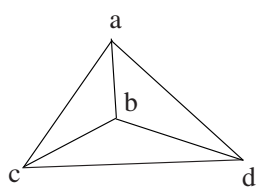

(b)

Fig. 2. (a) No geometric bistellar flip from edge $(a, b)$ to $(c, d)$ is possible because $(c, d)$ lies outside the quadrilateral $(a, b, c, d)$. However, edge $(a, b)$ is topologically flippable in the abstract triangulation given by the facets $(a, b, c),(a, b, d),(b, c, e)$, and $(b, d, e)$. (b) Edge $(a, b)$ is topologically unflippable because edge $(c, d)$ is already in the triangulation.

face flip, respectively. See Fig. 3. An edge is topologically unflippable if does not have exactly three incident faces or the face that would replace it is already in the complex. A face is topologically unflippable if the edge that would replace it is already in the complex. Again, in a geometric setting, a face can be geometrically unflippable even if it is topologically flippable. This occurs when there is a dihedral angle of an edge of the face which is reflex. See Fig. 4. An edge is geometrically unflippable if it is topologically unflippable or if it is on the boundary of the complex. There is one additional geometric possibility to consider: four coplanar points triangulated by an edge with four incident tetrahedra. This complication involves introducing the 4-to-4 flip or making a "general position assumption." It is not relevant topologically because it can be simulated with a sequence of other bistellar operations.

\section{An Unflippable Tetrahedralization, $U$ of $\mathbb{S}^{3}$}

We present a pure abstract tetrahedral complex, $U$, comprised of 16 vertices, 120 edges, 208 triangles, and 104 tetrahedra, having the following two properties:

1. The 1-skeleton of $U$ is the complete graph.

2. Each pair of vertices comprises four or more tetrahedra.

The first property guarantees that $U$ has neither a 2-to-3 flip nor a 4-to-1 flip. The second property guarantees that $U$ has no topological 3-to-2 flip. Thus, the only possible bistellar flip from $U$ is a 1-to-4 flip.
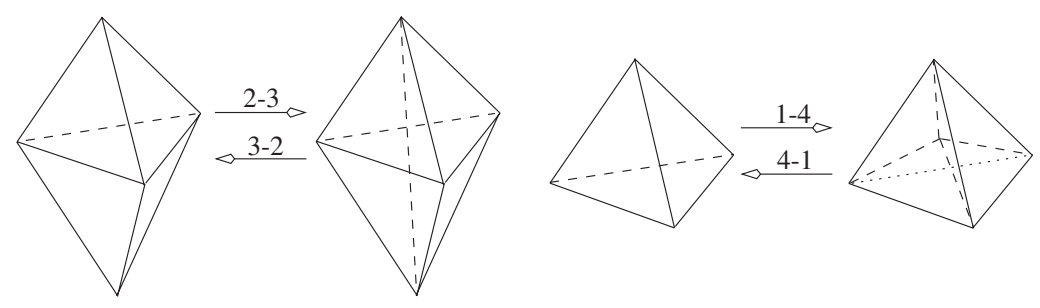

Fig. 3. Bistellar flips in three dimensions. 


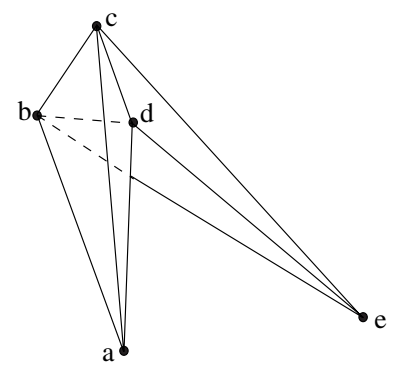

Fig. 4. Face $(b, c, d)$ is geometrically unflippable. If edge $(a, e)$ were part of the complex, $(b, c, d)$ would be topologically unflippable as well.

A computer search method borrowed from combinatorial design theory and used quite successfully to generate manifolds in Lutz's thesis [Lu1] was used to find $U$. The method uses orbits of vertex-transitive groups.

Theorem 1. The flip graph can be disconnected when $N$ is the set of all tetrahedralizations of $\mathbb{S}^{3}$ on $n$ nodes. Consider the following complex, $U$, with tetrahedra:

$\begin{array}{rrrrrrrrrrrrrrrrrrrr}0 & 1 & 8 & 9 & 1 & 2 & 9 & 10 & 2 & 3 & 10 & 11 & 3 & 4 & 11 & 12 & 4 & 5 & 12 & 13 \\ 5 & 6 & 13 & 14 & 6 & 7 & 14 & 15 & 0 & 7 & 8 & 15 & 0 & 1 & 8 & 10 & 1 & 2 & 9 & 11 \\ 2 & 3 & 10 & 12 & 3 & 4 & 11 & 13 & 4 & 5 & 12 & 14 & 5 & 6 & 13 & 15 & 0 & 6 & 7 & 14 \\ 1 & 7 & 8 & 15 & 0 & 2 & 8 & 9 & 1 & 3 & 9 & 10 & 2 & 4 & 10 & 11 & 3 & 5 & 11 & 12 \\ 4 & 6 & 12 & 13 & 5 & 7 & 13 & 14 & 6 & 8 & 14 & 15 & 0 & 7 & 9 & 15 & 0 & 1 & 4 & 9 \\ 1 & 2 & 5 & 10 & 2 & 3 & 6 & 11 & 3 & 4 & 7 & 12 & 4 & 5 & 8 & 13 & 5 & 6 & 9 & 14 \\ 6 & 7 & 10 & 15 & 0 & 7 & 8 & 11 & 1 & 8 & 9 & 12 & 2 & 9 & 10 & 13 & 3 & 10 & 11 & 14 \\ 4 & 11 & 12 & 15 & 0 & 5 & 12 & 13 & 1 & 6 & 13 & 14 & 2 & 7 & 14 & 15 & 0 & 3 & 8 & 15 \\ 0 & 1 & 4 & 6 & 1 & 2 & 5 & 7 & 2 & 3 & 6 & 8 & 3 & 4 & 7 & 9 & 4 & 5 & 8 & 10 \\ 5 & 6 & 9 & 11 & 6 & 7 & 10 & 12 & 7 & 8 & 11 & 13 & 8 & 9 & 12 & 14 & 9 & 10 & 13 & 15 \\ 0 & 10 & 11 & 14 & 1 & 11 & 12 & 15 & 0 & 2 & 12 & 13 & 1 & 3 & 13 & 14 & 2 & 4 & 14 & 15 \\ 0 & 3 & 5 & 15 & 0 & 1 & 6 & 14 & 1 & 2 & 7 & 15 & 0 & 2 & 3 & 8 & 1 & 3 & 4 & 9 \\ 2 & 4 & 5 & 10 & 3 & 5 & 6 & 11 & 4 & 6 & 7 & 12 & 5 & 7 & 8 & 13 & 6 & 8 & 9 & 14 \\ 7 & 9 & 10 & 15 & 0 & 8 & 10 & 11 & 1 & 9 & 11 & 12 & 2 & 10 & 12 & 13 & 3 & 11 & 13 & 14 \\ 4 & 12 & 14 & 15 & 0 & 5 & 13 & 15 & 0 & 1 & 10 & 14 & 1 & 2 & 11 & 15 & 0 & 2 & 3 & 12 \\ 1 & 3 & 4 & 13 & 2 & 4 & 5 & 14 & 3 & 5 & 6 & 15 & 0 & 4 & 6 & 7 & 1 & 5 & 7 & 8 \\ 2 & 6 & 8 & 9 & 3 & 7 & 9 & 10 & 4 & 8 & 10 & 11 & 5 & 9 & 11 & 12 & 6 & 10 & 12 & 13 \\ 7 & 11 & 13 & 14 & 8 & 12 & 14 & 15 & 0 & 9 & 13 & 15 & 0 & 2 & 9 & 13 & 1 & 3 & 10 & 14 \\ 2 & 4 & 11 & 15 & 0 & 3 & 5 & 12 & 1 & 4 & 6 & 13 & 2 & 5 & 7 & 14 & 3 & 6 & 8 & 15 \\ 0 & 4 & 7 & 9 & 1 & 5 & 8 & 10 & 2 & 6 & 9 & 11 & 3 & 7 & 10 & 12 & 4 & 8 & 11 & 13 \\ 5 & 9 & 12 & 14 & 6 & 10 & 13 & 15 & 0 & 7 & 11 & 14 & 1 & 8 & 12 & 15 & & & \end{array}$

One can see the 1-skeleton of $U$ is a complete graph and every edge is contained in four or more tetrahedra. To determine that $U$ is a tetrahedralization of $\mathbb{S}^{3}$, we considered guaranteed 3-sphere recognition algorithms such as those in [Th]. However, they appeared difficult to implement. Somewhat ironically, we used BISTELLAR, a flip-based simulated annealing program for shrinking a simplicial complex [Lu2]. Since $U$ is unflippable, we had to modify it by adding a few verticies and performing a few random 
2-to-3 flips. With this modification, BISTELLAR rapidly flipped $U$ down to a 4-simplex, thus proving $U$ is a tetrahedralization of $\mathbb{S}^{3}$ by Pachner's theorem.

We now show that tetrahedralizations of $\mathbb{S}^{3}$ with the properties listed in Section 3 cannot be realized as a tetrahedralization of any point set. To be precise about what we mean by geometrically realizable, we use the notion of a $d$-diagram, defined in [Zi].

Theorem 2. Tetrahedralizations of $\mathbb{S}^{3}$ whose 1-skeleton is the complete graph and every edge is contained in four or more tetrahedra do not have combinatorially equivalent 3-diagrams.

Proof. Let $U$ be a tetrahedralization of $\mathbb{S}^{3}$ with those properties and suppose $U$ has a combinatorially equivalent 3-diagram, call it $C$. Then the outside face of $C$ would be a tetrahedron $t$. We claim that there is a vertex $a$ of $t$ such that removing $a$ and all simplices incident upon it from $C$ would yield a convex 3-polytope with $n-1$ vertices. (Convexity follows because the 1-skeleton of $U$ is the complete graph.) This convex 3 -polytope would be tetrahedralized with no interior vertices. Now, each internal edge in the tetrahedralization of this polytope has four or more tetrahedra containing it. Further, there are $(n-1)(n-2) / 2$ edges in the tetrahedralized polytope, including the boundary edges. Therefore the tetrahedralization of the polytope is unflippable. However, this contradicts the results in [dLSU] which shows that any tetrahedralization of a convex polytope in $\mathbb{R}^{3}$ with no interior vertices always has flips.

\section{Conclusion}

We have shown that topologically unflippable tetrahedralizations of $\mathbb{S}^{3}$ whose 1-skeletons are the complete graph cannot resolve the flip-graph connectivity question for tetrahedralizations of point sets in $\mathbb{R}^{3}$. Nevertheless, it is not obvious that it is necessary that the 1-skeleton of a tetrahedralization of $\mathbb{S}^{3}$ be a complete graph to be topologically unflippable. Indeed, we have found topologically unflippable tetrahedralizations of other manifolds whose 1-skeletons are not the complete graph. It would be interesting to know if such tetrahedralizations exist for $\mathbb{S}^{3}$. If so, can they be geometrically realized in $\mathbb{R}^{3}$ ?

\section{Acknowledgements}

We thank the reviewers for their thoughtful remarks.

\section{References}

[ABS] A. Altshuler, J. Bokowski, and L. Steinberg. The classification of simplicial 3-spheres with nine vertices into polytopes and nonpolytopes. Discrete Math., 31:115-124, 1980.

[ACM] J. Ambjorn, M. Carfora, and A. Marzuoli. The Geometry of Dynamical Triangulations. Lecture Notes in Physics. Springer-Verlag, Berlin, 1997. 
[BL] A. Björner and F. Lutz. Simplicial manifolds, bistellar flips, and a 16-vertex triangulation of the Poincaré homology 3-sphere. Exposition. Math., 9:275-289, 2000.

[BS] L. J. Billeria and B. Sturmfels. Fiber polytopes. Ann. of Math., 135:527-545, 1992.

$\left[\mathrm{CDE}^{+}\right]$S.-W. Cheng, T. K. Dey, H. Edelsbrunner, M. A. Facello, and S.-H. Teng. Sliver exudation. In Proc. 15th Annu. ACM Sympos. Comput. Geom., pp. 1-13, 1999.

[De] O. Devillers. On deletion in a Delaunay triangulation. In Proc. 15th Annu. ACM Sympos. Comput. Geom., pp. 181-188, 1999.

[dL] J. A. De Loera. Triangulations of Polytopes and Computational Algebra. Ph.D. thesis, Applied Mathematics Department, Cornell University, 1995.

[dLSU] J. A. de Loera, F. Santos, and J. Urrutia. The number of geometric bistellar neighbors of a triangulation. Discrete Comput. Geom., 21(1):131-142, 1999.

[EPW] H. Edelsbrunner, F. P. Preparata, and D. B. West. Tetrahedralizing point sets in three dimensions. J. Symbolic Comput., 10(3-4):335-348, 1990.

[ES] H. Edelsbrunner and N. R. Shah. Incremental topological flipping works for regular triangulations. Algorithmica, 15:223-241, 1996.

[GKS] L. J. Guibas, D. E. Knuth, and M. Sharir. Randomized incremental construction of Delaunay and Voronoi diagrams. Algorithmica, 7:381-413, 1992.

[Jo] B. Joe. Three-dimensional triangulations from local transformations. SIAM J. Sci. Statist. Comput., 10(4):718-741, 1989

[La] C. L. Lawson. Software for $C^{1}$ surface interpolation. In J. R. Rice, editor, Mathematical Software III, pages 161-194. Academic Press, New York, 1977.

[Li] W. B. R. Lickorish. Simplicial moves on complexes and manifolds. Geom. Topol. Mono., 2:299-320, 1999.

[Lu1] F. Lutz. Triangulated Manifolds with Few Vertices and Vertex-Transitive Group Actions. Ph.D. thesis, Technische Universität, Berlin, 1999.

[Lu2] F. Lutz. Gap-program BISTELLAR. Available at http://www.math.tu-berlin.de/diskregeom/stellar/ BISTELLAR, 2000.

[Pa] U. Pachner. Konstruktionsmethoden und das kombinatorische homöomorphieproblem für triangulationen kompakter semilinearer mannigfaltigkeiten. Abh. Math. Sem. Univ. Hamburg, 57:69-86, 1987.

[Sa] F. Santos. A point configuration whose space of triangulations is disconnected. J. Amer. Math. Soc., 13(3):611-637, 2000.

[Th] A. Thompson. Thin position and the recognition problem for the 3-sphere. Math Res. Lett., 1:613630, 1994.

[Wa] K. Wagner. Bemekungen zum vierfarbenproblem. J. Deut. Math., 46:26-32, 1936.

[Zi] G. M. Ziegler. Lectures on Polytopes. Graduate Text in Mathematics. Springer-Verlag, Berlin, 1995.

Received February 28, 2002, and in revised form December 1, 2003. Online publication July 5, 2004. 